\title{
The Symplectic Method in Polar Coordinates for Linear Viscoelastic Materials
}

\author{
W.X. Zhang* and Y. Bai \\ School of Civil Engineering and Architecture, Henan University of Technology, Zhengzhou, Henan, P.R. China
}

\begin{abstract}
In this study, the symplectic method is applied to a two-dimensional annular-sector viscoelastic domain under the polar coordinate system. By applying variable separation approach, all fundamental solutions are derived in analytical form. Furthermore, using the method of variable substitution, lateral conditions are transformed into finding a particular solution for the governing equations, and this particular solution is derived with the use of eigensolution expansion. In the numerical example, the boundary condition problem is discussed in detail to analyze the stress response of viscoelastic solids.
\end{abstract}

Keywords: Polar coordinate, symplectic method, viscoelastic.

\section{INTRODUCTION}

Analysis of viscoelastic materials is proved to be more complex than elastic ones since viscoelastic materials show elastic and viscous response simultaneously, when external forces are loaded [1]. In recent years, the research in viscoelasticity has become an important topic, and a large sum of reports can be found in literature [2,3]. It is well known that the stress-strain relation of this kind of materials is time-dependent, and therefore various numerical computational methods are inevitably taken into account in these research reports, especially the widely used finite element method. Using this approach, Khan and Muliana studied the thermal and mechanical effects, and presented a numerical approach to analyze coupled thermo-visco-elastic responses [4]. In Cao and Chen's work [5], a theoretical analysis based on the finite element is investigated for solving engineering problems related with viscoelastic composite. The boundary element is another important approach in viscoelastic computations. By constructing a time formulation with the use of the reciprocity theory, Cezario et al. derived a set of concise general solutions for plane viscoelastic problems [6]. Moreover, Zéhil and Gavin analyzed three-dimensional problems, and proposed a new boundary element for incompressible viscoelastic layers [7]. Obviously, the boundary element approach provides an attractive idea since the dimension of the problem can be reduced [6].

On the other hand, the symplectic mechanics based on Hamiltonian formulation provide an alternative and efficient way for solving solid and fluid mechanics including viscoelastic problems [8]. In contrast to the well-known semi-inverse method, the symplectic approach takes dual variables (displacement and stress components) as the basic variables, and hence the difficulty for solving high order

*Address correspondence to this author at the School of Civil Engineering and Architecture, Henan University of Technology, Zhengzhou, Henan, P.R. China; Tel: 18623717351; E-mail: weixiang_zh@163.com differential equations in the traditional methods is bypassed. Unlike the above traditional methods, the dual variables (displacement and stress components) are selected as fundamental variables in the symplectic system, and accordingly the order of partial differential equations is reduced. During the past two decades, the symplectic method has been introduced into various fields of engineering applications successfully. In elasticity, a great amount of symplectic techniques can be found in literature including the work on free vibration, thin plate bending, and circular cylindrical problems [9-11]. Moreover, the symplectic elasticity approach has been recently extended to problems with complex material properties and structures. $\mathrm{Xu}$ et al. presented an analytical solution for three-dimensional transversely isotropic piezoelectric media in Hamiltonian form [12]. Li and Yao (2011) derived a general solution for twodimensional magnetoelectroelastic solids [13]. Regarding viscoelastic materials, the symplectic method cannot be used directly due to the existence of the energy dissipation. Using the integral transformation, however, the original problem is described as energy conservative one, and thus the symplectic method can be applied.

In this paper, the quasi-static viscoelastic problem in an annular-sector domain is analyzed under the symplectic system. By employing the variable separation approach, all general solutions is analytically obtained in terms of polar coordinates. Since the eigenvectors has the character of concise analytical form, one can easily get the corresponding time domain expressions. Compared with the traditional symplectic methods, the proposed approach is very convenient for viscoelastic problems with annular-sector domain, especially for those with viscoelastic crack. As an illustration, we discussed a typical boundary condition problem in the numerical example, and the corresponding stress distribution is presented to verify the proposed symplectic model and also to show local effects near the ends of the annular-sector domain. 


\section{THE FUNDAMENTAL PROBLEM IN THE SYMPLECTIC SYSTEM}

As Fig. (1) shows, the polar coordinate $(r, \alpha)$ is selected to consider a plain annular-sector domain $(a \leq r \leq b,-\theta \leq \alpha \leq \theta)$, where $\mathrm{r}$-axis is in the direction of the radius of the sector, and the origin is located at the center point. The Cartesian coordinates $(x, y)$ are used as a referential system.

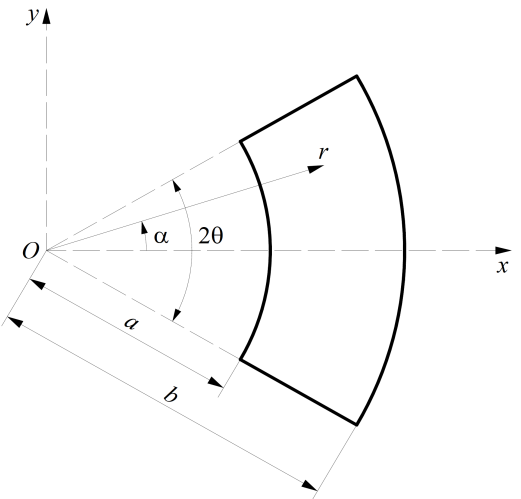

Fig. (1). The sketch map of the annular-sector in the polar coordinates.

The material is supposed to be time-dependent linear viscoelastic solid. Its constitutive relation can be described through integral equation [14]:

$\sigma_{i j}(t)=\delta_{i j} \int_{-\infty}^{t} \lambda(t-\tau) \frac{\partial \varepsilon_{k k}(\tau)}{\partial \tau} d \tau+2 \int_{-\infty}^{t} \mu(t-\tau) \frac{\partial \varepsilon_{i j}(\tau)}{\partial \tau} d \tau$

where $\sigma_{i j}$ and $\varepsilon_{i j}$ are stress and strain components, $\varepsilon_{k k}$ is the volumetric strain, and $\mu(t)$ and $\lambda(t)$ are relaxation moduli. The corresponding Laplace domain description of Eq. (1) is:

$$
\bar{\sigma}_{i j}=\bar{\lambda}(s) \bar{\varepsilon}_{k k}(s) \delta_{i j}+2 \bar{\mu}(s) \bar{\varepsilon}_{i j}(s)
$$

in which an over bar on a modulus represents the Laplace transformation, and $\mathrm{s}$ is the complex-valued transform parameter. In general, the shear modulus can be expressed by a micro-model including parallel spring-dashpots (Fig. 2). The model leads to:

$$
\mu(t)=\mu_{\infty}+\sum_{i} \mu_{i} \mathrm{e}^{-p_{i} t}
$$

where $p_{i}=\mu_{i} / \eta_{i}$. The Laplace transform of Eq. (3) is:

$$
\bar{\mu}(s)=\frac{\mu_{\infty}}{s}+\sum_{i} \frac{\mu_{i}}{s+p_{i}}
$$

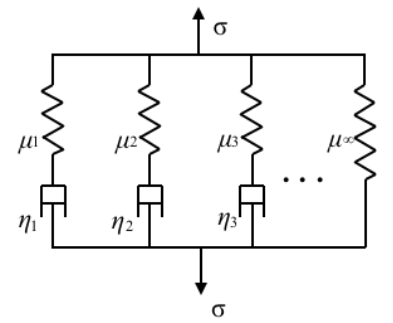

Fig. (2). The viscoelastic model.
Generally, the Poisson's ratio $v$ is also time-dependent, but compared with other moduli, the dependence is much weaker. In this paper, we consider the Poisson's ratio as a constant modulus.

Without considering body forces, the Lagrange function can be described as:

$\bar{L}=\frac{r}{2}\left(\bar{\sigma}_{r} \bar{\varepsilon}_{r}+\bar{\sigma}_{r \alpha} \bar{\varepsilon}_{r \alpha}+\bar{\sigma}_{\alpha} \bar{\varepsilon}_{\alpha}\right)$

In the polar coordinate system, the symplectic method can also be applied. However, a new axis, $\xi=\ln r$, must be introduced. Under the new coordinates $(\xi, \alpha)$, the total potential energy is expressed by displacement variables as:

$$
\begin{aligned}
\Phi= & \int_{\ln a}^{\ln b} \int_{-\theta}^{\theta}\left\{\frac{\bar{\mu}}{2}\left(\frac{\partial \bar{u}}{\partial \alpha}+\dot{\bar{v}}-\bar{v}\right)+\frac{\bar{\mu}}{1-v}\left[\dot{\bar{u}}^{2}+\left(\bar{u}+\frac{\partial \bar{v}}{\partial \alpha}\right)^{2}\right.\right. \\
& \left.\left.+2 v \dot{\bar{u}}\left(\bar{u}+\frac{\partial \bar{v}}{\partial \alpha}\right)\right]\right\} d \xi d \alpha
\end{aligned}
$$

where a dot over a variable donates the differentiation with respect to $\xi$. From minimum potential energy principle, we have

$\delta \Phi=0$

For the convenience of discussion, we introduce the displacements vector and its dual vector:

$\overline{\mathbf{Q}}=\left[\frac{\bar{u}}{v}\right], \quad \overline{\mathbf{P}}=\frac{\partial \bar{L}}{\partial \dot{\overline{\mathbf{Q}}}}=\left[\begin{array}{l}\bar{S}_{r} \\ \bar{S}_{r \alpha}\end{array}\right]$

where

$$
\bar{S}_{r \alpha}=\bar{\mu}\left(\dot{\bar{v}}-\bar{v}+\frac{\partial \bar{u}}{\partial \alpha}\right)=r \bar{\sigma}_{r \alpha},
$$

$\bar{S}_{r}=\frac{2 \bar{\mu}}{1-v}\left[v\left(\bar{u}+\frac{\partial \bar{v}}{\partial \alpha}\right)+\dot{\bar{u}}\right]=r \bar{\sigma}_{r}$.

For energy conservative problems, the variational method can be applied. Using Eq. (7), we have the dual equations in the Hamiltonian system:

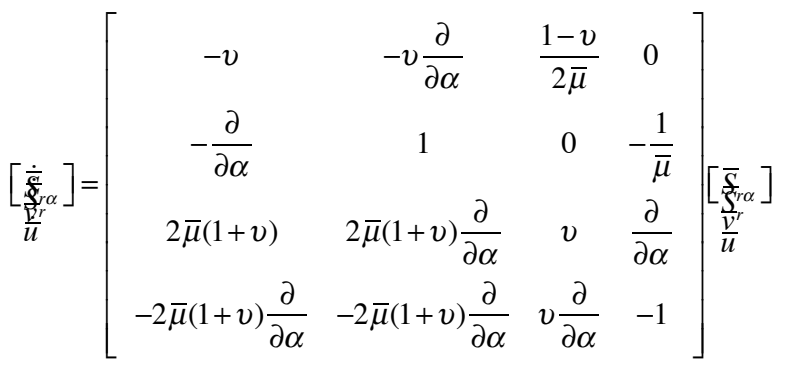

For brevity, Eq. (9) is simplified as:

$\bar{\psi}=\mathbf{H} \bar{\psi}$

The other stress component is:

$\bar{S}_{\alpha}=r \bar{\sigma}_{\alpha}=2(1+v) \bar{\mu}\left(\bar{u}+\frac{\partial \bar{v}}{\partial \alpha}\right)+v \bar{S}_{r}$. 


\section{FUNDAMENTAL SOLUTIONS}

Suppose the lateral boundary is displacement free, that is:

$\bar{\sigma}_{\alpha}=\bar{\sigma}_{r \alpha}=0 \quad(\alpha= \pm \theta)$

Using the variable separation approach, we can assume the final solution

$\bar{\psi}(\xi, \alpha)=\bar{\omega}(\xi) \overline{\mathbf{Y}}(\alpha)$

in which $\bar{\omega}(\xi)=e^{\kappa \xi}$, and $\overline{\mathbf{Y}}(\alpha)$ satisfy

$\mathbf{H} \overline{\mathbf{Y}}(\alpha)=\kappa \overline{\mathbf{Y}}(\alpha)$

where $\kappa$ is an eigenvalue, and $\overline{\mathbf{Y}}$ is its corresponding eigenvector:

$\overline{\mathbf{Y}}=[\overline{\mathbf{q}}]=\left[\bar{q}_{1}, \bar{q}_{2}, \bar{p}_{1}, \bar{p}_{2}\right]^{\mathrm{T}}$

The characteristic equation of Eq. (13) is:

$\operatorname{det}\left[\begin{array}{cccc}\kappa+v & \lambda v & (v-1) /(2 \bar{\mu}) & 0 \\ -\lambda & 1-\kappa & 0 & 1 / \bar{\mu} \\ 2 \bar{\mu}(1+v) & 2 \bar{\mu}(1+v) \lambda & v-\kappa & -\lambda \\ 2 \bar{\mu}(1+v) \lambda & 2 \bar{\mu}(1+v) \lambda^{2} & \lambda v & 1+\kappa\end{array}\right]=0$

\subsection{The Case of $\kappa \neq 0$}

Four different characteristic values can be found from Eq. (15), and therefore we describe the general solution as:

$\overline{\mathbf{Y}}=\left[\begin{array}{c}c_{11} \cos (1+\kappa) \alpha+c_{12} \sin (1+\kappa) \alpha+c_{13} \cos (1-\kappa) \alpha+c_{14} \sin (1-\kappa) \alpha \\ c_{21} \sin (1+\kappa) \alpha+c_{22} \cos (1+\kappa) \alpha+c_{23} \sin (1-\kappa) \alpha+c_{24} \cos (1-\kappa) \alpha \\ c_{31} \cos (1+\kappa) \alpha+c_{32} \sin (1+\kappa) \alpha+c_{33} \cos (1-\kappa) \alpha+c_{34} \sin (1-\kappa) \alpha \\ c_{41} \sin (1+\kappa) \alpha+c_{42} \cos (1+\kappa) \alpha+c_{43} \sin (1-\kappa) \alpha+c_{44} \cos (1-\kappa) \alpha\end{array}\right]$

Because solution (16) must satisfy the lateral boundary condition (11), the constants $c_{j k}$ are dependent. As a result, only four constants are independent, and accordingly the solutions can be rewritten as:

$$
\begin{aligned}
\overline{\mathbf{Y}}= & {\left[\begin{array}{c}
c_{1} \beta_{11} \cos (1+\kappa) \alpha+c_{2} \beta_{12} \cos (1-\kappa) \alpha \\
c_{1} \beta_{21} \sin (1+\kappa) \alpha+c_{2} \beta_{22} \sin (1-\kappa) \alpha \\
c_{1} \beta_{31} \cos (1+\kappa) \alpha+c_{2} \beta_{32} \cos (1-\kappa) \alpha \\
c_{1} \beta_{41} \sin (1+\kappa) \alpha+c_{2} \beta_{42} \sin (1-\kappa) \alpha
\end{array}\right] } \\
& +\left[\begin{array}{c}
c_{3} \beta_{13} \sin (1+\kappa) \alpha+c_{4} \beta_{14} \sin (1-\kappa) \alpha \\
c_{3} \beta_{23} \cos (1+\kappa) \alpha+c_{4} \beta_{24} \cos (1-\kappa) \alpha \\
c_{3} \beta_{33} \sin (1+\kappa) \alpha+c_{4} \beta_{34} \sin (1-\kappa) \alpha \\
c_{3} \beta_{43} \cos (1+\kappa) \alpha+c_{4} \beta_{44} \cos (1-\kappa) \alpha
\end{array}\right]
\end{aligned}
$$

where $\beta_{11}=\beta_{12}=\beta_{13}=\beta_{14}=-\beta_{21}=\beta_{23}=1, \beta_{22}=-\beta_{24}=(v$ $-\kappa-\kappa v-3) /(3-v-\kappa-\kappa v), \beta_{31}=\beta_{33}=-\beta_{41}=\beta_{43}=2 \bar{\mu} \kappa$, $\beta_{32}=\beta_{34}=-2 \bar{\mu} \kappa(1+v)(3-\kappa) /(3-v-\kappa-\kappa v)$

$\beta_{42}=-\beta_{44}=-2 \bar{\mu} \kappa(1+v)(1-\kappa) /(3-v-\kappa-\kappa v)$. The two terms in the right of Eq. (17) represent solutions for symmetrical problems and anti-symmetrical ones respectively. To determine the eigenvalues, substituting the solutions (17) into Eq. (11), we get:

$\left[\begin{array}{cl}-\frac{1}{1+v} \cos (1+\kappa) \theta & \frac{1+\kappa}{3-v-\kappa-\kappa v} \cos (1-\kappa) \theta \\ -\frac{1}{1+v} \sin (1+\kappa) \theta & \frac{1-\kappa}{3-v-\kappa-\kappa v} \sin (1-\kappa) \theta\end{array}\right]\left[\begin{array}{l}c_{1} \\ c_{2}\end{array}\right]=\mathbf{0}(18)$

for the symmetric solutions, and

$$
\left[\begin{array}{cc}
-\frac{1}{1+v} \sin (1+\kappa) \theta & \frac{1+\kappa}{3-v-\kappa-\kappa v} \sin (1-\kappa) \theta \\
\frac{1}{1+v} \cos (1+\kappa) \theta & -\frac{1-\kappa}{3-v-\kappa-\kappa v} \cos (1-\kappa) \theta
\end{array}\right]\left[\begin{array}{l}
c_{3} \\
c_{4}
\end{array}\right]=\mathbf{0}
$$

for the anti-symmetric ones. Due to the existence of non-zero eigenvectors, the coefficient determinants of Eqs. (18) and (19) must be zeros. Accordingly, the eigenvalue equations can be established.

\subsection{The Case of $\kappa=0$}

Solutions for this case are usually called zero eigenvectors. The governing equation is:

$$
\mathbf{H} \overline{\mathbf{Y}}(\alpha)=0
$$

By solving Eq. (20) directly, we get two fundamental solutions:

$$
\begin{aligned}
& \overline{\mathbf{Y}}_{1}^{(0)}=\left[\begin{array}{llll}
\cos \alpha & -\sin \alpha & 0 & 0
\end{array}\right]^{\mathrm{T}} \\
& \overline{\mathbf{Y}}_{2}^{(0)}=\left[\begin{array}{llll}
\sin \alpha & \cos \alpha & 0 & 0
\end{array}\right]^{\mathrm{T}}
\end{aligned}
$$

Their geometrical meanings are rigid translations in $x$ and $y$ directions, respectively. Besides Eqs. (21) and (22), there are corresponding Jordan form solutions. The governing equation is:

$$
\mathbf{H} \overline{\mathbf{Y}}_{n}^{(j)}=\overline{\mathbf{Y}}_{n}^{(j-1)}
$$

Two Jordan form solutions can be found according to Eq. (23):

$$
\begin{aligned}
& \overline{\mathbf{Y}}_{1}^{(1)}=\left[\begin{array}{c}
(1-v) \alpha \sin \alpha \\
(1-v) \alpha \cos \alpha-(1+v) \sin \alpha \\
4 \bar{\mu}(1+v) \cos \alpha \\
0
\end{array}\right] \\
& \overline{\mathbf{Y}}_{2}^{(1)}=\left[\begin{array}{c}
(v-1) \alpha \cos \alpha \\
(1-v) \alpha \sin \alpha+(1+v) \cos \alpha \\
4 \bar{\mu}(1+v) \sin \alpha \\
0
\end{array}\right]
\end{aligned}
$$

They are the solutions of concentrated forces at the origin along the vertical and horizontal directions, respectively. It can be proved that there exist no other Jordan form solutions. Since the eigenvectors has the character of concise analytical 
form, one can easily get the corresponding time domain expressions with the use of the inverse integral transform.

\section{LATERAL BOUNDARY CONDITIONS}

The above mentioned fundamental solutions are derived under the condition that the lateral boundary is in homogeneous form. For non-homogeneous cases, we can transform the non-homogeneous conditions into homogeneous ones simply by using the method of variable substitution. As an example, we suppose:

$$
\begin{aligned}
& \bar{S}_{\alpha}=\bar{S}_{\sigma}^{ \pm} \\
& \bar{S}_{r \alpha}=\bar{S}_{r \sigma}^{ \pm}
\end{aligned} \quad(\alpha= \pm \theta)
$$

Eq. (26) can be expressed by the fundamental variables of the symplectic system as:

$$
\begin{aligned}
& \bar{S}_{r \alpha}=\bar{S}_{r \sigma}^{ \pm} \\
& 2 \bar{\mu}(1+v)\left(\bar{u}+\frac{\partial \bar{v}}{\partial \alpha}\right)+v \bar{S}_{r}=\bar{S}_{\sigma}^{ \pm} \quad(\alpha= \pm \theta)
\end{aligned}
$$

Boundary conditions (27) can be expressed in homogeneous form under new variables:

$\overline{\psi^{\prime}}=\bar{\psi}-\overline{\psi^{\prime \prime}}$

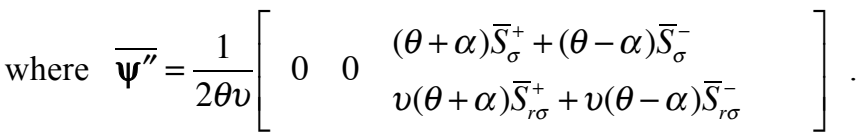
One can verify that boundary conditions (27) are transformed into homogeneous ones. Yet the dual equation (13) becomes non-homogeneous:

$$
\overline{\psi^{\prime}}=\mathbf{H} \overline{\psi^{\prime}}+\overline{\mathbf{h}}^{\prime}
$$

where the non-homogeneous term can be obtained by Eq. (13):

$$
\overline{\mathbf{h}}^{\prime}=\mathbf{H} \overline{\psi^{\prime \prime}}-\bar{\psi}^{\prime \prime}
$$

Therefore, the lateral boundary condition problem is reduced by finding a particular solution of governing equations.

In fact, the complete solution includes a certain combination of general solutions and a particular solution.

\section{NUMERICAL ANALYSIS}

As an application of the symplectic method, numerical examples are given here. In this section, the first three terms of the relaxation modulus (4) are used. The viscoelastic parameters of the solid are given as: $\mu_{1}=\mu_{2}=5 \mu_{\infty}$, $\eta_{1}=\eta_{2}=\eta$, and $v=0.3$. The geometrical models shown in Fig. (1) are selected as: $b=2 a$, and $\theta=\pi / 12$. As Fig. (3) shows, the boundary conditions are:

$$
\begin{array}{ll}
u=v=0 & (r=a) \\
\sigma_{r}=\sigma_{r \alpha}=0 & (r=b) \\
\sigma_{\alpha}=P, \sigma_{r \alpha}=0 & (\alpha= \pm \theta)
\end{array}
$$

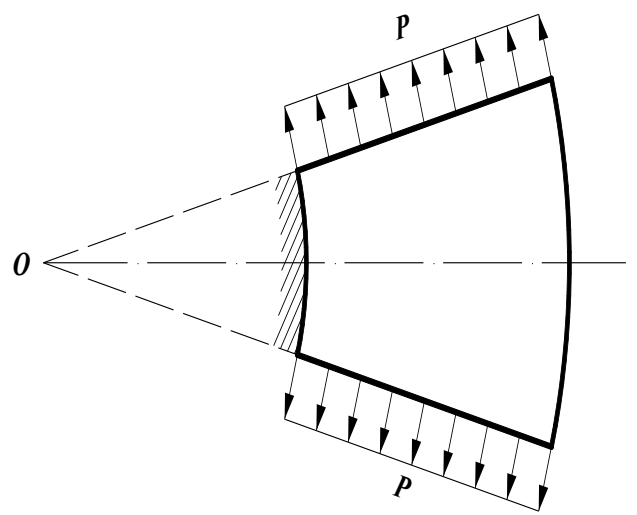

Fig. (3). The geometrical model.

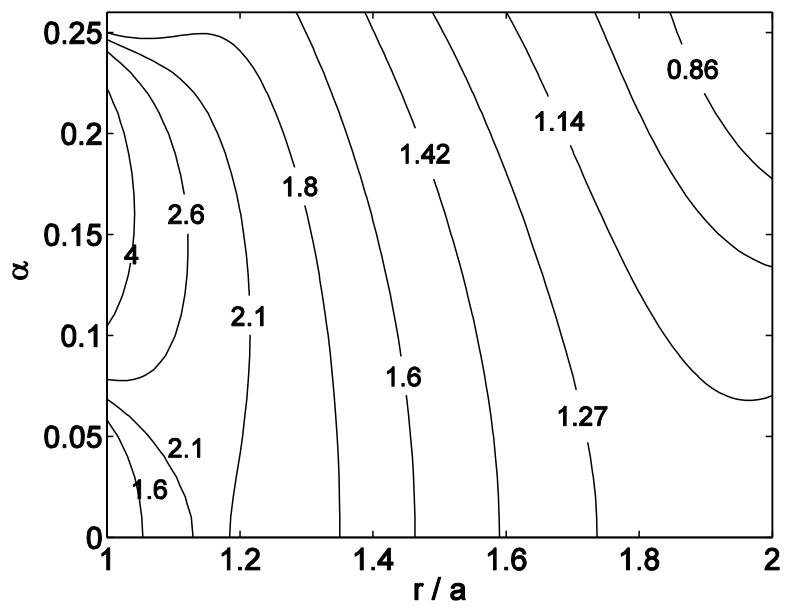

Fig. (4). Stress $\sigma_{\alpha} / P$ distribution.

Based on the symplectic theory of this research, we firstly transform the non-homogeneous conditions $(\alpha= \pm \theta)$ into homogeneous form using the method of variable substitution. Then, considering circular boundary conditions $(r=a, b)$, we established linear equations about the coefficients of the eigenvector. Thus, the components of $\sigma_{r}$, $\sigma_{\alpha}$ and $\sigma_{r \alpha}$ are obtained numerically. According to the results, $\sigma_{\alpha}$ plays the most important role among the stress, and its distribution is given in Fig. (4). It is clear that stress concentrations appear near the clamped boundary due to the displacement constraints, and the concentration effect attenuates dramatically with the radial direction. The results of the example well agree with the famous Saint-Venant principle about boundary effects.

\section{CONCLUSION}

The polar coordinate symplectic method is applied for viscoelastic materials in the Laplace domain, and all the general eigensolutions are derived analytically. With the use of the adjoint symplectic relations, solutions of various boundary condition problems are conveniently obtained by combinations of the fundamental eigensolutions. Numerical examples show that stress concentrations effects decrease at rapid speed with the distance from the clamped boundary. Compared with the traditional symplectic methods, the 
proposed approach is more suitable for engineering computations of solids and structures in annular-sector domains, and further researches of elastic or viscoelastic cracks are highly expected due to the use of the polar coordinate system.

\section{CONFLICT OF INTEREST}

The author confirms that this article content has no conflict of interest.

\section{ACKNOWLEDGEMENTS}

Declared none.

\section{REFERENCES}

Barbagallo M, Finnveden S. A self-adjoint variational principle for anisotropic viscoelastic Biot's equations. Int J Eng Sci 2013; 63: 71-83.

[2] Nguyen ST, Jeannin L, Dormieux L, Renard F. Fracturing of viscoelastic geomaterials and application to sedimentary layered rocks. Mech Res Commun 2013; 49: 50-56.

[3] Lei Y, Adhikari S, Friswell MI. Vibration of nonlocal KelvinVoigt viscoelastic damped Timoshenko beams. Int J Eng Sci 2013; 66-67: 1-13.

[4] Khan KA, Muliana AH. Fully coupled heat conduction and deformation analyses of visco-elastic solids. Mech TimeDependent Mat 2012; 16: 461-89.
[5] Cao YP, Chen KL. Theoretical and computational modelling of instrumented indentation of viscoelastic composites. Mech TimeDepend Mat 2012; 16: 1-18.

[6] Cezario F, Santiago JAF, Oliveira RF. Two-dimensional version of Sternberg and Al-Khozaie fundamental solution for viscoelastic analysis using the boundary element method. Eng Analy Bound Elements 2011; 35: 836-44.

[7] Zéhil GP, Gavin HP. Three-dimensional boundary element formulation of an incompressible viscoelastic layer of finite thickness applied to the rolling resistance of a rigid sphere. Int $\mathbf{J}$ Solids Struct 2013; 50: 833-42.

[8] Zhong WX. On the reciprocal theorem and adjoint symplectic orthogonal relation. Acta Mechanica Sinica 1992; 24: 432-7.

[9] Lim CW. Symplectic elasticity approach for free vibration of rectangular plates. Adv Vib Eng 2010; 9: 159-63.

[10] Lim CW, Cui S, Yao WA. On new symplectic elasticity approach for exact bending solutions of rectangular thin plates with two opposite sides simply supported, Int J Solids Struct 2007; 44: 5396411.

[11] Tarn JQ, Tseng WD, Chang HH. A circular elastic cylinder under its own weight. Int J Solids Struct 2007; 46:2886-96.

[12] Xu XS, Leung AYT, Gu Q, Yang H, Zheng JJ. 3D symplectic expansion for piezoelectric media. Int J Num Meth Eng 2008; 74 : 1848-71.

[13] Li XC, Yao WA. Symplectic analytical solutions for the magnetoelectroelastic solids plane problem in rectangular domain. J Appl Math 2011; 2011: 1-15.

[14] Sawanta S, Muliana A. A thermo-mechanical viscoelastic analysis of orthotropic materials. Composit Struct 2008; 63: 1-72. 